\title{
UNIVERSAL ECO ENVIRONMENTAL DRONE IONIZER EQUIPMENT FOR DRONES USING VERTICAL PROPELLERS
}

\author{
Gheorghe Silviu Ionut ${ }^{1}$, Negrea Catalin Stefan ${ }^{1}$, Angelescu Dorin ${ }^{1}$ Robert Mangher ${ }^{2}$, Vlad Mangher ${ }^{3}$ \\ ${ }^{1}$ National Institute of Research and Development in Mechatronics and Measurement Technique, \\ INCDMTM - Bucharest, Romania \\ ${ }^{2}$ S.C. MDM Standard S.R.L., Romania \\ ${ }^{3}$ Faculty of Mechatronics, University "Politehnica" of Bucharest, Romania \\ Emails: silviu1_silviu1@yahoo.com, catalin.negrea95@gmail.com,dorinnirod@yahoo.com , \\ robert mangher@mdmstandard.ro, vlad robert01@yahoo.com
}

\begin{abstract}
Started as a collaboration between the CERMISO center of INCDMTM and MDM Standard S.R.L. the idea of an ECO Environmental Drone Ionizer Device (EEDI) has started in the spring of 2021 when the predicted future number of drones and application implied was in visible grow. In fact if the drone activity is increasing, we substantiated the idea of a device that, regardless of the type or mission of the drone, helps to depollute travel environment i.e. the air. It became obvious that the huge amount of air displaced by the drone rotors can be ionized through a specialized device. The device itself, for the moment named ECO Environmental Drone Ionizer (EEDI), must have few extremely important features:

- Must be able to ionize the whole mass of the air columns passing through the rotors;

- Must not affect the drone activity, regardless of the types of missions;

- Must not decrease the lift and/or the flight autonomy more than a few percent;

- Must be able to adapt on any type of drone with vertical propellers;

- Must not affect the drone features and performance;

- It must be completely safe for both the equipment and the operator.

In fact if each drone mission use an EDDI device, then the drone is resolving two targets in the same time, the mission itself and also purifies a large amount of air that passes through its propellers. So, the more drones there are in the air, the more air will be purified. And we think this would be an interesting step alongside the current global environmental regeneration movement.
\end{abstract}

Keywords: Eco-drones, Air-purification, Air-ionizer, Ionizing antena, Eco-friendly.

\section{Introduction}

In nature, the process of air ionization consists in the production of negative ions, also known as "air vitamins", to balance the amount of positive ions, resulting from pollution, for example. Numerous researches so far have shown that ionization of the air makes an important contribution in neutralizing pathogens, such as bacteria and viruses, removing dust or volatile chemicals, smoke, leaving the air cleaner and, implicitly, healthier.

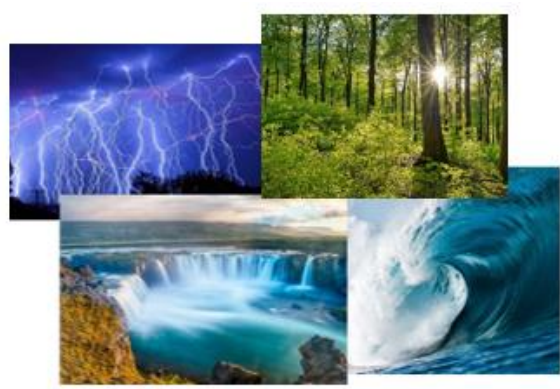

- ultraviolet (UV) rays from the Sun;

- electrical discharges during storms;

- waterfalls;

- waves on the shores of oceans and seas;

- plants, especially during the growth process.

The air we breathe contains gaseous components, liquids, solid particles, microorganisms, molecular compounds and fractions of it all. Ions are molecules or atoms with electric charge. The ions in the air component are formed when a molecule or atom of oxygen, nitrogen or other gases receives enough energy, under the action of ultraviolet rays, cosmic rays, soil radioactivity, lightning, the "Lenard effect" (occurs when shore waves break, during heavy rains or waterfalls and consists of spraying and dispersing water droplets), in order to release an electron.

Negative ions are those that gain an electron on the last layer (the electron shell), while positive ions lose an electron. Hence the electrical nature of the ions and the chemical reactivity. Negative or positive ions in the air are invisible, odorless, tasteless molecules. The amounts of ions differ, depending on 
the environment. Thus, in areas where the air is oxygenated, there is a lot of vegetation, near water, beaches, waterfalls, negative ions predominate. Instead, in polluted areas, in hermetically sealed spaces, where there is no proper air circulation, the amount of positive ions is higher. Negative ions were discovered 100 years ago and have since been the subject of numerous studies that have questioned the effects they have on the environment in which we live. It should be noted that not all research has led to definite conclusions and that some of them have even sparked controversy among scientists. However, experts from the National Institutes of Health and WebMD point out certain effects that ionization can have on the body, noting that further studies are needed in this regard. Thus, negative ions could contribute to:

- reducing the effects of depression and stress once we inhale them, the negative ions reach our circulatory system, where they can produce certain biochemical reactions that increase serotonin levels; this can lead to a reduction in anxiety or depression, a decrease in stress and an increase in energy throughout the day;

- improving the manifestations of allergies some research has shown that ionization can help those who have symptoms of an allergy to dust, fungal spores and molds or other allergens;

- elimination of small suspended particles - this is a complex group of pollutants that differ in terms of size, shape, composition and source that produces them; carbon emissions from engines, lead particles, rubber, road traffic dust, construction and other industrial waste, pollen and other soil particles, are all examples of such pollutants; large particles are usually filtered naturally by the body through the nose and throat; small particles can get into the airways and lungs, causing health problems; ionization helps to better absorb them by air purifiers. When natural sources of negative ions are missing, artificial ionization, which is possible with modern equipment, replaces the benefits found in nature (when walking in the woods, by the sea or the ocean, next to a cascade).

Specifically, artificial ionization emits negative oxygen ions, which are trapped by dust, allergens, and airborne particles. The latter become heavier and are aspirated more easily. Basically, a large amount of negative ions can restore the freshness of the air, thus contributing to a better and to less polluted air environment.

\section{Drones}

The drone is a UAV (Unmanned Aerial Vehicle) device capable of flying without being piloted by a person, which was originally used only for military purposes for the recognition of objectives and the bombing of strategic targets.
Drones have become increasingly popular with regular users as manufacturers have launched models for commercial use at low prices. As with the Internet, drones have moved beyond the military's exclusive sphere and, with some important changes, have come to be produced for commercial purposes. Currently, drones are also used for professional purposes for high-altitude filming of sporting events or concerts, but also by regular users who have discovered a new "toy" to spend time with. In fact, if military drones can be compared to airplanes, civilian drones are significantly smaller and less efficient, but are affordable for a diverse range of people.

Anyway, in the last years the drones seems to have an exponential evolution being implied in most of the human activities in most all domains. As predicted by specialists, the number of drones will increase the same way as predicted for all classical airborne transport at a rate over 35\% each 10 years. The European Commission estimates that, by 2035, the European drone sector:

- will directly provide jobs for over 100,000 people

- will have an economic impact of over EUR 10 billion per year, mainly in the field of services

As the use of drones expands, so will the need to find a balance between their advantages and the problems they generate. For example, unmanned aerial vehicles can provide added value when used for data collection and interpretation in different sectors of the economy. But drones can also create problems in terms of data protection, privacy, noise and $\mathrm{CO} 2$ emissions.

\section{The Premises}

Started as a collaboration between the CERMISO center of INCDMTM and MDM Standard S.R.L. the idea of an ECO Environmental Drone Ionizer Device (EEDI) has started in the spring of 2021 when the predicted future number of drones and application implied was in visible grow. In fact if the drone activity is increasing, we substantiated the idea of a device that, regardless of the type or mission of the drone, helps to depollute travel environment i.e. the air. It became obvious that the huge amount of air displaced by the drone rotors can be ionized through a specialized device. The device itself, for the moment named ECO Environmental Drone Ionizer (EEDI), must have few extremely important features:

- Must be able to ionize the whole mass of the air columns passing through the rotors;

- Must not affect the drone activity, regardless of the types of missions;

- Must not decrease the lift and/or the flight autonomy more than a few percent;

- Must be able to adapt on any type of drone with vertical propellers; 
- Must not affect the drone features and performance;

It must be completely safe for both the equipment and the operator.

In fact if each drone mission use an EDDI device, then the drone is resolving two targets in the same time, the mission itself and also purifies a large amount of air that passes through its propellers. So, the more drones there are in the air, the more air will be purified. And we think this would be an interesting step alongside the current global environmental regeneration movement.

\section{Tests and Preliminary Results of the Project}

The EEDI device was to be powered by the main drone battery and must also have minimal weight in order to not interfere with the features and performance of the flight equipment. We have taken into consideration an high voltage generator (negative with regard to earth) and passive antennas. Antennas must be protected from accidental touch, in order not to put the operator life in danger. Antennas must be interconnected and placed beneath each air column generated by the propellers. The air column passing through the antenna is ionized and released into the environment.

We have preferred the high voltage generator with passive antennas for the extremely low consumption of such equipment. We activate the antennas with the air column generated continuous by the propellers which in our case is the activator of the ionization process.

The high voltage generator is constructed around a DC-AC converter step up module and a voltage multiplier element connected to the passive antenna as shown in Figure 1.

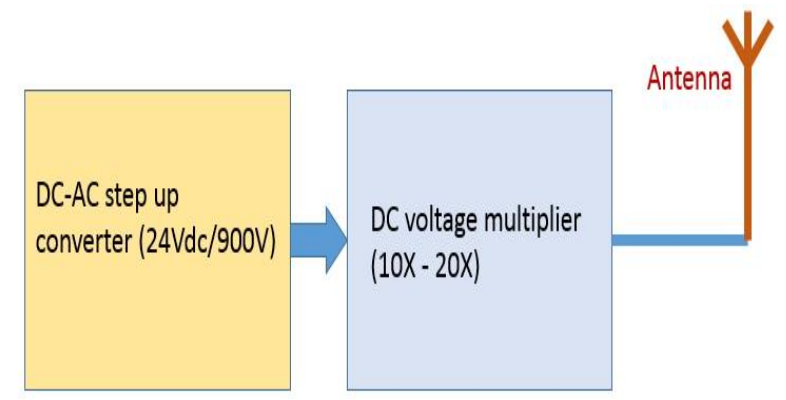

Figure 1: General diagram of EEDI device

The step up converter transform the voltage from the drone's power supply and using a local oscillator transform it into AC voltage at a first multiplied rate of 37 times. The result is an approximatively $900 \mathrm{~V}$ AC that is injected in the Dc voltage multiplier.

At a rate that can be made somewhere around 10 - 20 times we can obtain between 4500V and 9000V injected into the passive antenna. The antenna must be carefully dimensioned and designed to assure the best ionization rate and minimal interference with the drone lift parameters.

In Figure 2 it is shown the DC-AC step up converter. The main idea was to minimize the weight of the device, so we adopt one of the classical versions of a CMOS oscillator using CD4093, a quad 2 input NAND in order to obtain a perfect rectangular signal form that is forwarded to transistor T1. T1 has,

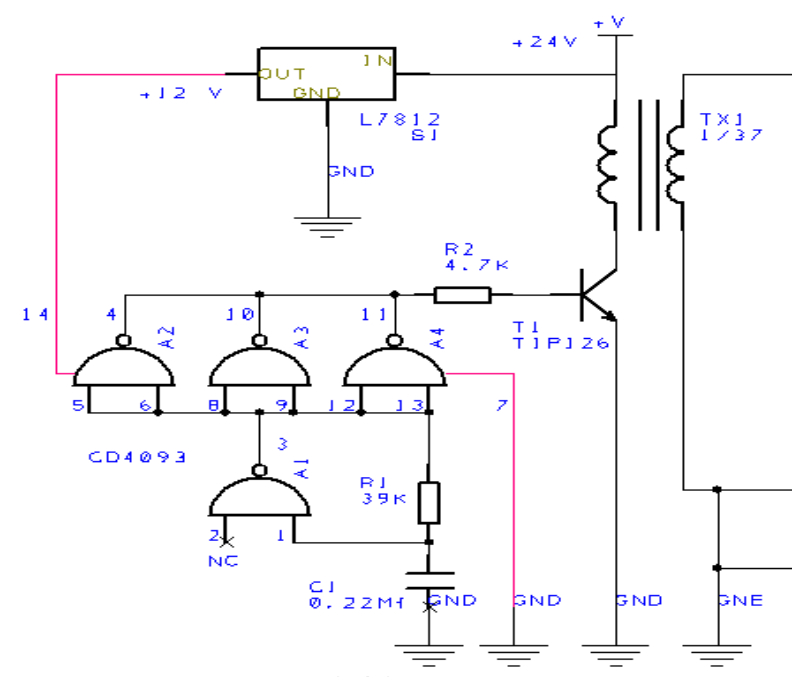

Figure 2: DC-AC step up converter

as a load, the primary winding of the TX1 transformer. Considering a ratio $1 / 37$ between primary and secondary of TXJ the $24 \mathrm{AC}$ is multiplied to approx. 900 volts. It was also necessary to reduce the $24 \mathrm{~V}$ to $12 \mathrm{~V}$ in order to power the CMOS CD4093 circuit using a positive voltage regulator L7812 (S1 in Figure 2). The $900 \mathrm{~V}$ exit is injected into a 20 stage voltage multiplier as shown in Figure 3.

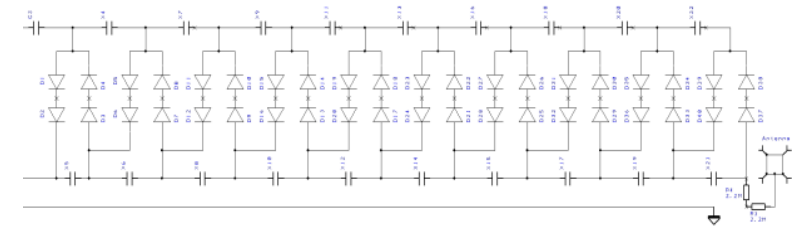

Figure 3: The voltage multiplier

Finally at the exit of the voltage multiplier (it is used the half-wave series multiplier, also called the Villard cascade) it was obtained the DC high voltage. Each additional stage of two diodes and two capacitors increases the output voltage by twice the peak AC supply voltage the exit of the multiplier is in our case around $9 \mathrm{kV}$ DC output. The $9 \mathrm{kV}$ exit is applied to the ionization antenna. For the moment test have been made with those parameters, as the project is still in progress, but, if necessary, it will possible to increase the number of multiplier stages.

Finally, the complete design is shown in Figure 4. The total weight of the device is (in actual tests, with 
the 20 stages multipliers) around 55 grams (most of the TXJ transformer) which is, at least for the moment, a negligible load for the drone. So in Figure 4 we can see the complete electric diagram of the device.

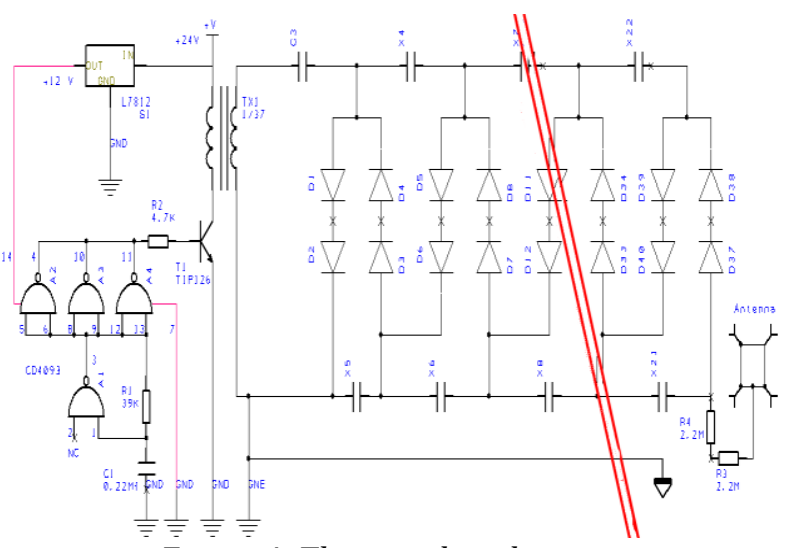

Figure 4: The complete diagram

In order to avoid involuntary dangerous current discharges the connection between the antenna and the high tension source is made thru two $2.2 \mathrm{Mohms}$ resistors.

Another solution for the high tension device is to use „20KV High Frequency High Voltage Transformer Ignition Coil Inverter Driver Board" available on the market composed by a DC -AC convertor using the NE555 CI as a DC-AC convertor as presented in Figure 5.
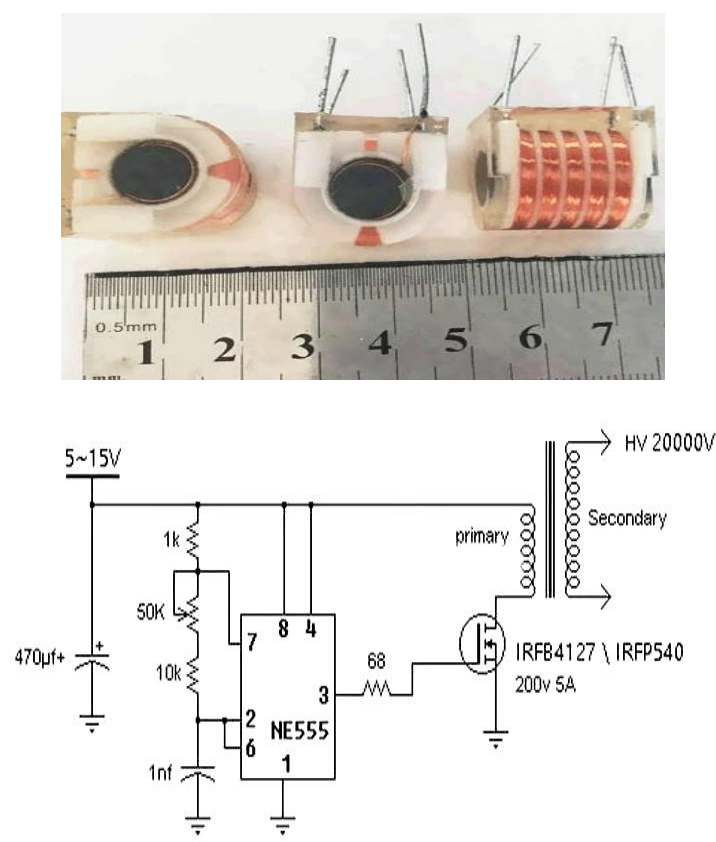

Figure 5: The 20KV ignition coil and the driver

Using the same principle, this time the rectangle signal generator is the NE555 timer IC and the IRFB4127 transistor. The kit can be found on line for prices as low as 5-7 USD. There is also possible to change the frequency of the rectangular signal using the $50 \mathrm{~K}$ pot similar to the design from Figure 5 where it must be changed the values of R1 and C1. The design from Figure 5 obtains a much higher value of the high voltage directly on the secondary of the transformer. Both transformers can be exchanged from first and second drivers and a multiplier similar to the one in Figure 3 can also be added to the secondary of the transformers from Figure 5 (minimum 2 stages or more if necessary).

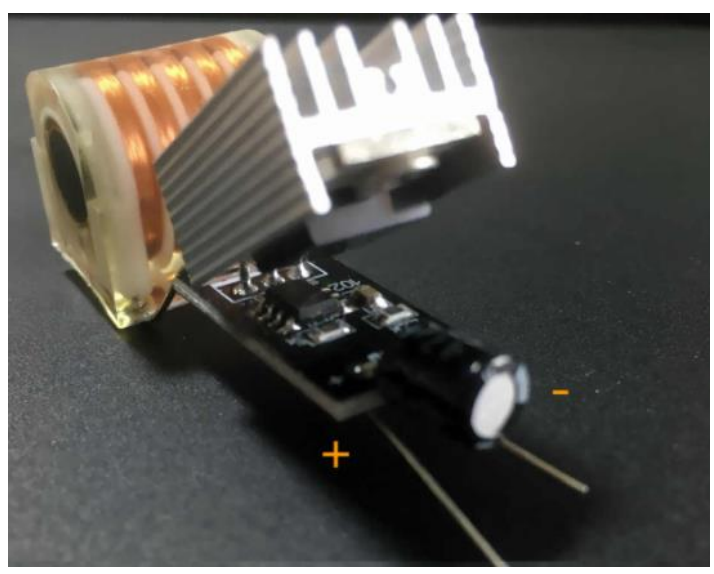

Figure 6: The driver and the High Voltage Transformer presented in Figure 5 (without voltage multiplier) weight at about 30 grams.

The ionization antenna must be executed extremely rigid, and isolated according to the final voltage exit taking into consideration that no accidental discharges must occur between the drone body and the antenna. Generally it is considered that an electric discharge can occur in plain air at a $1 \mathrm{~mm}$ for $1 \mathrm{KV}$. Tests have demonstrated that it is better to consider a $2 \mathrm{~mm}$ per $1 \mathrm{KV}$ as a sure montage distance.

The antennas must be placed under the propellers and must have a diameter as close as possible to their length, or if possible, a little bigger in order to make the most of the columns of air that pull the ions out. There were made tests using various ionization antenna configurations and both drivers as presented in Figure 4 and 5. Both financially and functional the Figure 5 design seems to be optimal (with a 2 stages voltage multiplier added). A slightly better consumption can be obtained using the rectangle generator from Figure 4 with the transformer from Figure 5. The current consumption must be optimized considering a better adaptation of the final transistor by finding its better commutation transition according to the driver and the load $(100 \%$ commutation transition must be achieved for the minimal current consumption) in the final phase of the project.

Several forms of the ionization antenna were tested in order to determine a final coherent form of it, trying to find the best configuration as ions quantity and structure resistance. Until now (being a project in progress) we have tested the following configuration of ionizing antenna as presented in Figure 7. The tests were made into the CERTIM 
center clean room (total air volume $100 \mathrm{~m}^{3}$ ) using a stationary $45 \mathrm{~W}$ ventilator with an air flow of $900 \mathrm{~m}^{2}$ / hour (and the room hermetically closed).
The grade of ionization was measured quantitative using a negative ion detector device patented by Vincent Vollono and Tony van Roon (Figure 7).

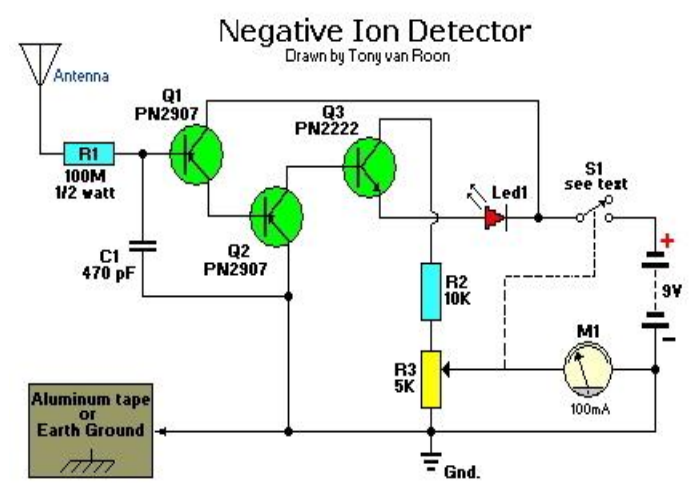

Figure 7: Negative Ion Detector (all rights reserved to Vincent Vollono and Tony van Roon)

The detector was placed in opposite diagonal of the ionizing antenna. The time between the start of the ventilator and the moment when the LED lights up is timed (strong ionization occur). After each type of antenna was tested the clean room was 1 hour ventilated to reset the ion concentration. Although laborious tests gave us extremely useful information about the impact of the antenna configuration and the amount of ions emitted. All antennas were made with copper wire in $1 \mathrm{~mm}$ diameter soldered, united with tin bath, into forms.

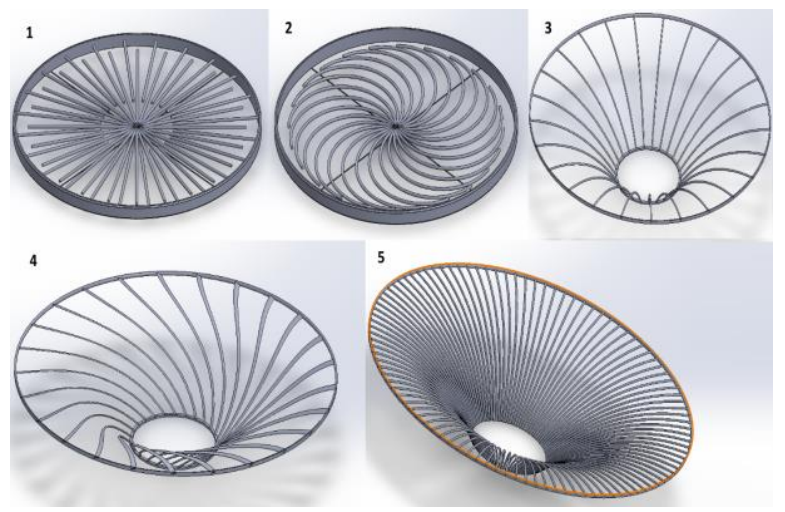

Figure 8: Types of ionizing antenna tested

The measured results are centralized in Table 1

\begin{tabular}{|c|c|c|}
\hline $\begin{array}{c}\text { Antenna } \\
\text { Type }\end{array}$ & $\begin{array}{c}\text { Ionization } \\
\text { Time }\end{array}$ & $\begin{array}{c}\text { Order } \\
\text { Number }\end{array}$ \\
\hline 1 & $5 \min 32 \mathrm{sec}$ & 3 \\
\hline 2 & $5 \min 47 \mathrm{sec}$ & 4 \\
\hline 3 & $6 \min 01 \mathrm{sec}$ & 5 \\
\hline 4 & $4 \mathrm{~min} 37 \mathrm{sec}$ & 2 \\
\hline 5 & $3 \min 46 \mathrm{sec}$ & 1 \\
\hline
\end{tabular}

We have made two types of structures generally speaking, one being straight (1,3 and 5) and the others being rotational in the same direction of rotation as the air column (4 and 2). It is thus confirmed first of all that the ionization is directly proportional to the number of spokes, which was to be expected. Secondly, however, there is an improved behavior in rotational models, especially in number 4 , given that the number of spokes in this case is 25 compared to 100 of the number 5 . But, a large number of spokes negatively influences the vortex air flow which leads to the loss of propeller efficiency. As a result we have to improve antenna number 4 changing the number of spikes from 25 to 50.

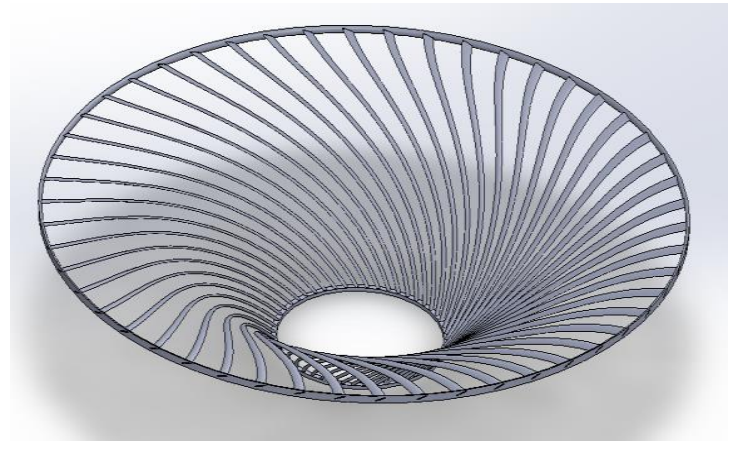

Figure 9: Antenna no.6 the improved test version of the no. 4 antenna (50spikes instead of 25).

Test confirmed that the ionization time decreased to 3 minutes and 52 seconds and moreover the impact to the air flow being minimal.

More test will be necessary to obtain the optimal ionization antenna, including new structures of inner spikes network, especially with many pointed terminals. 


\section{What is to be Done}

As the above results demonstrate, the scope of the project seems possible to be accomplished, but more research must be done. The next steps in the project are expected to be the following:

- More tests regarding new ionization antennas with a network of multiple pointed terminals;

- Improvement of the ionization detector in order to obtain a much precise indication of the real amount of ions concentration into the hermetic chamber;

- Realization of a universal antenna mounting system under the drone motors;

- Development of a system for determining the electrical conductivity of the air around the drone able to eliminate possible accidental discharges of the potential difference from the antenna to the drone body;

- Final test with the heavy load drone of CERMISO center (DJI Inspire 2 Standard Combo)

- The final results of the project will be subject of one or more patents.
The progress of this project will be the subject of other articles that the authors will present in this journal.

\section{References}

[1] United States Environmental Protection Agency, What are ionizers and other ozone generating air cleaners?

[2] https://www.epa.gov/indoor-air-qualityiaq/what-are-ionizers-and-other-ozonegenerating-air-cleaners

[3] Vincent Vollono and Tony van Roon, Built this negative Ion Detector, https://www.learningelectronics.net/VA3AVR/ci $\underline{\mathrm{rc} / \mathrm{hv} / \text { niondet/niondet.html }}$

[4] Wikipedia, Air Ionizer, https://en.wikipedia.org/wiki/Air ioniser

[5] Healthline, The Effect of Negative Ions, https://www.healthline.com/health/negativeions 